\title{
Stereotactic Body Radiotherapy in Patients with Lung Oligometastases from Colorectal Cancer
}

\author{
FRANCESCO PASQUALETTI ${ }^{1}$, SABRINA MONTRONE $^{1}$, CATERINA VIVALDI $^{2}$, MARGHERITA ZANI $^{3}$, \\ DAVID FEDELE ${ }^{3}$, LORENZO FORNARO ${ }^{2}$, GIUSEPPE PASQUALETTI ${ }^{4}$, LISA SALVATORE ${ }^{2}$, \\ BRUNO MANFREDI ${ }^{1}$, CONCETTA LALISCIA $^{1}$, GABRIELE CORAGGIO $^{1}$, \\ ALESSANDRA GONNELLI ${ }^{1}$, FOTIOS LOUPAKIS ${ }^{2}$, GIANLUCA MASI ${ }^{2}$, \\ ALDO SAINATO $^{1}$, FABIO MONZANI ${ }^{4}$, ALFREDO FALCONE $^{2}$ and FABIOLA PAIAR ${ }^{1}$ \\ ${ }^{1}$ Department of Clinical and Translational Sciences, \\ Division of Radiation Oncology, University of Pisa, Pisa, Italy; \\ ${ }^{2}$ Department of Clinical and Translational Sciences, Division of Medical Oncology, University of Pisa, Pisa, Italy; \\ ${ }^{3}$ Casa di Cura San Rossore, Division of Radiation Oncology, Pisa, Italy; \\ ${ }^{4}$ Department of Experimental and Clinical Medicine, Division of Geriatric Unit, University of Pisa, Pisa, Italy
}

\begin{abstract}
Aim: To assess the role of stereotactic body radiotherapy (SBRT) in pulmonary metastases from colorectal cancer (CRC). Patients and Methods: Thirty-three consecutive patients with pulmonary metastases from CRC who received SBRT were included in the analysis. The primary endpoints were local and systemic progression-free survival, a secondary endpoint was the safety profile of SBRT. Results: A total of 56 lesions were treated with SBRT. A single nodule was treated in 15 patients, two in 13 and three in five. The radiotherapy dose and the adopted fractionations were 24-27 Gy as a single fraction for 40 lesions and 27-42 Gy in three fractions (2-3 times a week) for the other 16 lesions. After a median follow-up of 22.8 months (range =1.3-45.7 months), the median progressionfree survival of the irradiated sites was 13.4 months. Conclusion: SBRT can be considered as local therapy in patients with lung metastases from CRC.
\end{abstract}

Colorectal cancer (CRC) is the third most common cancer diagnosed worldwide, as a matter of fact it accounts for around $10 \%$ of all total solid tumors and more than $1,300,000$ new recorded cases in 2015 (1). Despite the progresses achieved in early diagnosis and locoregional therapies, the onset of lung metastasis is expected to occur in $5-16 \%$ of patients with CRC

Correspondence to: Francesco Pasqualetti, MD, Ph.D., Department of Clinical and Translational Sciences, Division of Radiation Oncology, University of Pisa, Via Roma 67, 56123, Pisa, Italy. Tel: +39050993456 , e-mail: f.pasqualetti@ao-pisa.toscana.it/francep24@hotmail.com

Key Words: Stereotactic body radiotherapy, colorectal cancer, oligometastasic cancer, lung metastases.
$(1,2)$. After the diagnosis of pulmonary metastatic disease, systemic therapy represents the treatment cornerstone, improving survival and quality of life $(3,4)$. However, in selected patients with a limited burden of disease, considered as oligometastatic patients (5), survival can be enhanced even by surgical resection of metastases $(3,6)$. An analysis of surgical series, even if most studies were conducted in a small number of patients and in a retrospective manner, shows that when complete resection of the metastatic lesions is achievable, the 5-year overall survival ranges from $25 \%$ to $70 \%(7,8)$. In recent years, many studies of stereotactic body radiotherapy (SBRT) with an ablative intent have been conducted in patients with metastatic cancer, including CRC (9-12). Considering its safety and the chances of achieving the control of treated lesions, in many patients with metastatic solid tumors, SBRT could be considered as an alternative to surgery (13-16). Despite the studies carried out in patients with oligometastatic cancer, little is known about SBRT for patients with lung metastasis from CRC cancer, especially considering advances in radiotherapy, e.g. volumetric-modulated arc therapy (VMAT) and image-guided radiotherapy (IGRT). By combining V-MAT and IGRT, high doses of ionizing radiation can be delivered to very small areas (such as metastasis) excluding tissues not grossly involved with tumor (lung parenchyma).

This retrospective mono-institutional analysis aimed to evaluate the activity and the safety of SBRT in a cohort of 33 consecutive patients with lung metastases from CRC.

\section{Patients and Methods}

Study design. We report a retrospective analysis of 33 consecutive patients affected by lung metastases from CRC and treated with SBRT between May 2011 and March 2015 at the Pisa University 
Hospital. Patient clinical data and treatment related data were retrieved from the Pisa University Hospital database, the study . was approved by the Comitato Etico di Area Vasta Nord Ovest (CEAVNO), Pisa, Italy, (CEAVNO prot. n. 1235). All treated patients gave their written consent for SBRT.

Treatment procedure. SBRT treatments were performed using a Varian True Beam ${ }^{\circledR}$ platform (Varian Medical System, Inc., Palo Alto City, California, USA) and 6-MV photons with flattening filter-free beams. RapidArc ${ }^{\circledR}$ system (Varian Medical System, Inc., Palo Alto City, California, USA) was used for treatment planning. Axial computed tomographic (CT) images were obtained using a Light Speed RT 16-slice simulator (General Electric Company, GE HealthCare, Milan, Italy). All patients were immobilized in the supine position with a long whole-body vacuum bag as support. 3D CT images were acquired corresponding to the three phases of the respiratory cycle (conventional free-breathing, end-inhalation and end-exhalation). The CT image slice was set at $1.25 \mathrm{~mm}$. Target lesions, gross tumor volume (GTV), were identified using CT lung window setting (1600-400 Hounsfield units) on the three CT image fusions. Clinical Target Volumes (CTVs) were made fusing the GTVs obtained for the three different respiratory phases. CTV were made fusing the three GTV. Planning target volume consisted of an isotropic $3 \mathrm{~mm}$ expansion of the clinical target volume. Pulmonary lesions were treated with $24-26$ Gy as a single fraction or using a hypofractionated schedule (27-42 Gy in three fractions). The choice of dose and fractionation depended on tumor volume and location (close proximity to critical structures such as heart, great vessels, main bronchus and esophagus). The delivered dose were prescribed to the periphery of the target. Before each fraction, a cone-beam CT was performed for each patient's set-up. Task Group 101 of the American Association of Physicists in Medicine constraints were used to avoid toxicity of the organs at risk (17). Toxicity was recorded using the Common Terminology Criteria for Adverse Events, version 4.0 (18).

Before SBRT, all patients were staged with fluorodeoxyglucose positron emission computerized tomography (PET/CT) or total body CT scan with contrast agent. In order to assess the local response and systemic disease, CT scanning or total body PET/CT were performed 6-8 weeks after the end of SBRT. Thereafter, every 3-4 months, patients underwent follow-up examinations with total body $\mathrm{CT}$ scanning or total body PET/CT, carcinoembryonic antigen (CEA) and carbohydrate antigen 19-9 (CA19.9) assessment; clinical oncologists or medical oncologists carried out clinical examinations. After SBRT, further systemic therapies were not administered until disease progression.

Statistical analyses. The primary objective of this analysis was the time to progression of treated lesions. Lesion progression was measured by response evaluation criteria in solid tumor (RECIST) (19). The secondary objective was progression-free survival with respect to regions outside the irradiated site and SBRT safety profile. All the intervals were measured from SBRT to event occurrence. Kaplan-Meier method and Cox proportional hazard models were used to analyze time-to-event variables and factors associated with primary and secondary objectives, respectively.

\section{Results}

Patients. Patient characteristics are shown in Table I. Twenty-five patients were males and 8 females; the median age at the time of SBRT was 67 years (range=31-88 years). Diagnosis of primary CRC was histological confirmed in all
Table I. Patient characteristics.

\begin{tabular}{lrc}
\hline Characteristic & $\mathrm{N}$ & $\%$ \\
\hline Gender & & \\
$\quad$ Male & 25 & 75.7 \\
$\quad$ Female & 8 & 24.3 \\
Metastases treated perpatient & 15 & \\
$\quad 1$ & 13 & 45.5 \\
2 & 5 & 39.4 \\
3 & & 15.1 \\
Site of lung metastases & 29 & 51.8 \\
$\quad$ Inferior/medium lobe & 27 & 48.2 \\
$\quad$ Superior lobe & & \\
Primary site of cancer & 19 & 57.6 \\
$\quad$ Rectum & 14 & 42.4 \\
$\quad$ Colon & & \\
Fractionation & 40 & 71.4 \\
$\quad$ Fraction & 16 & 28.6 \\
$\quad 3$ Fractions &
\end{tabular}

patients and the site of primary tumor was rectum and colon in 19 and 14 patients, respectively. Seven patients had synchronous metastasis at the time of diagnosis of CRC (three patients had lung metastasis). Thirty-two patients underwent surgery at the primary site of disease (rectum or colon), while one patient did not because of age and comorbidity, and only palliative radiotherapy to the rectum was performed as local therapy.

A total of 56 active lesions in 33 consecutive patients were treated. At the time of analysis the median follow-up was 22.8 (range $=1.3-45.7$ ) months. Delivered radiotherapy dose was 24-27 Gy as a single fraction for 40 lesions and 27-42 Gy delivered in three fractions (2-3 times a week) for 16 lesions. A single nodule was treated in 15 patients, two in 13 and three in five. The median planning target volume was $21.45 \mathrm{cc}$ (range $=6-156 \mathrm{cc}$ ). The median gross tumor volume was $2.3 \mathrm{cc}$ (range=0.6-68 cc). Twenty-nine irradiated lesions were localized to the inferior or medium lobe, while 27 to the superior lobe. Local relapses were recorded in 23 lesions (41.1\%); lesion control rate, defined by CT scan or PET/CT, at 6, 12 and 18 months was $87.8 \%, 62.0 \%$ and $30.0 \%$, respectively. Figure 2 shows two CT scans performed before and after SBRT. The median time to progression of the irradiated sites was 13.4 months, while outside the irradiated site it was 6.8 months (Figure 1).

Severe toxicities were not observed; only one grade 1 pneumonitis was recorded.

\section{Discussion}

Despite the lack of results derived from prospective randomized trials and the different endpoints adopted, local 

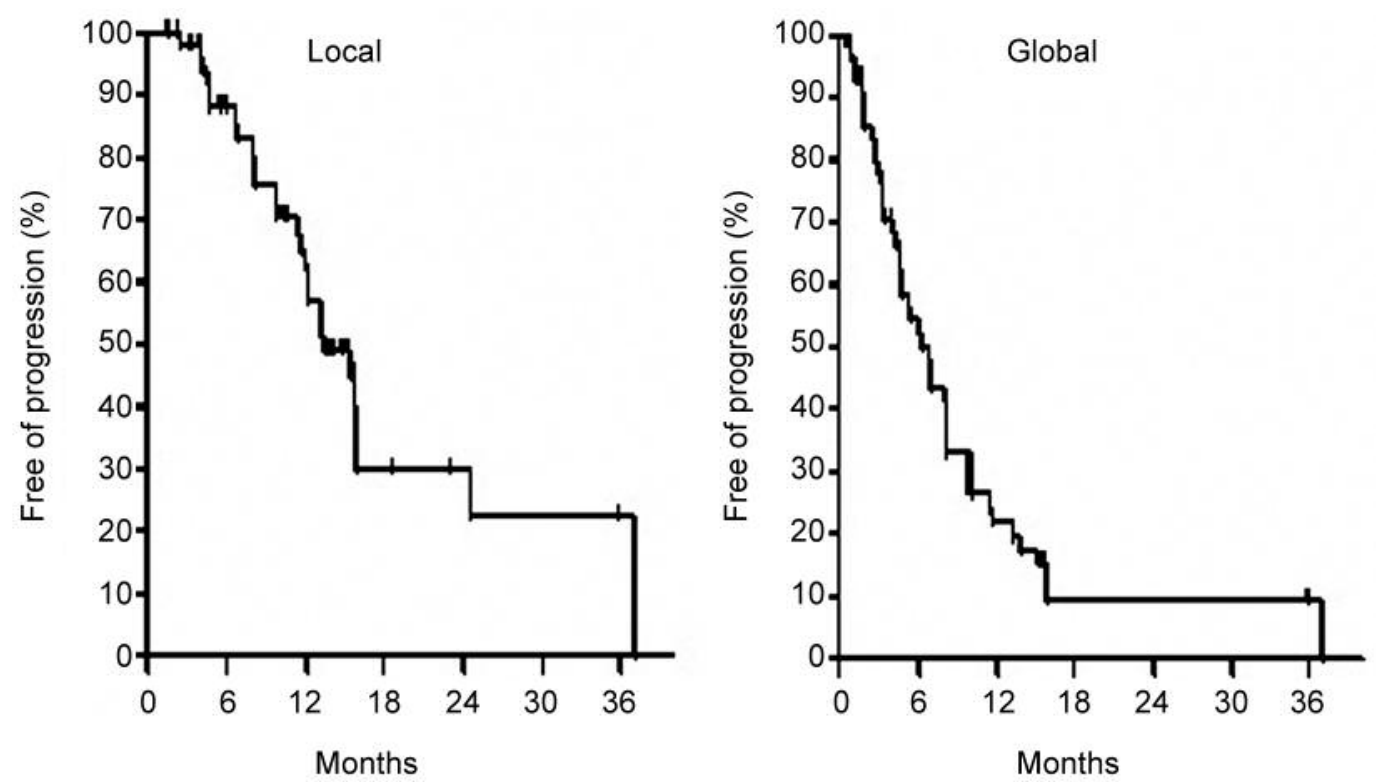

Figure 1. Local and global control after stereotactic body radiotherapy.
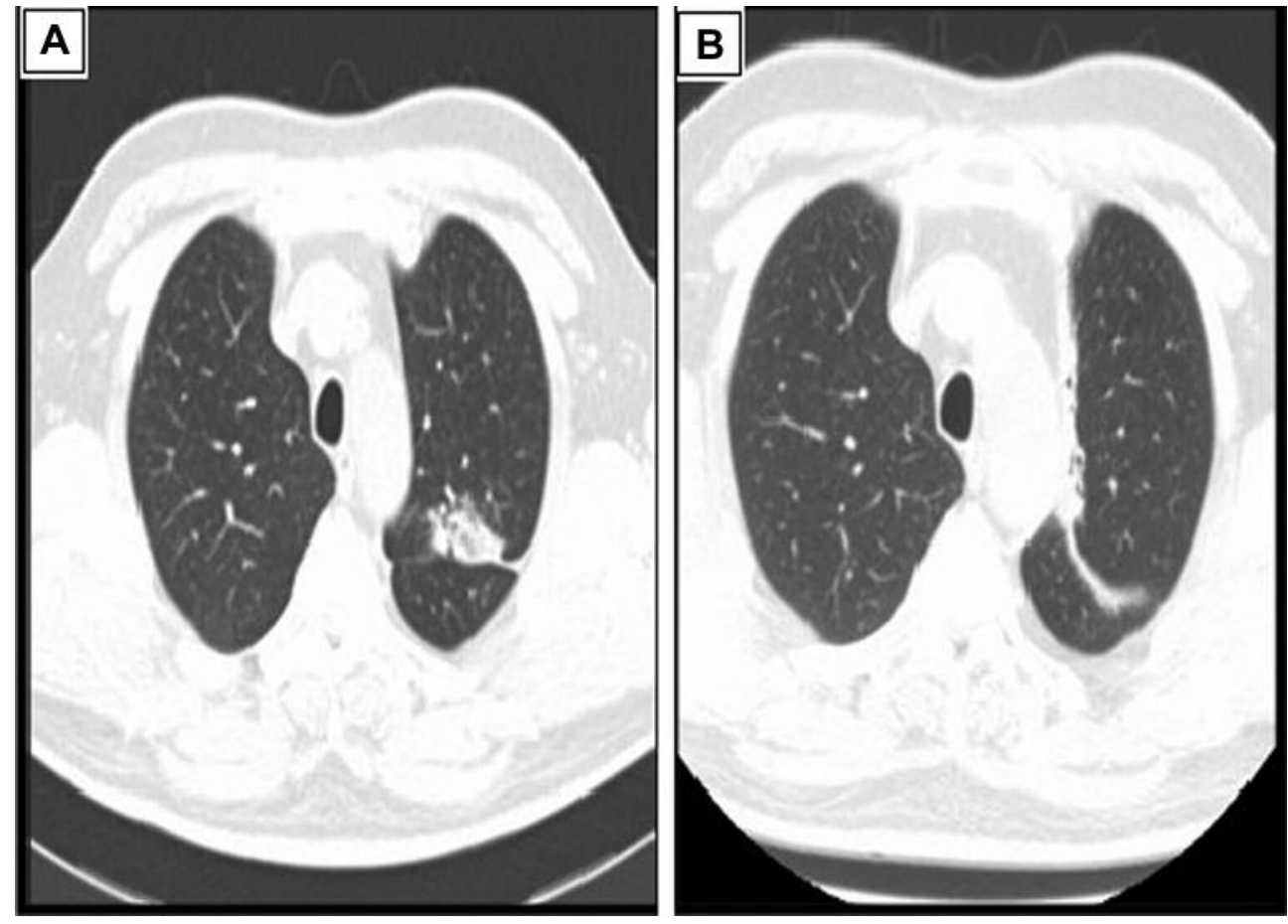

Figure 2. Computed tomography performed before $(A)$ and after $(B)$ stereotactic body radiotherapy showed disease control.

treatments as salvage therapy in patients with oligometastatic solid tumors represent an increasingly considered approach, mainly in frail patients, sometimes as an alternative to salvage systemic therapy or with the intent of postponing the beginning (14-16). Considering the wide range of doses and techniques adopted, and the absence of patient selection, prior experiences conducted using SBRT in patients with pulmonary metastases from CRC did not produce conclusive findings, 
neither, for lesions or disease control. Filippi et al., after studying 25 patients treated from 2004 to 2014 with a median follow-up of 20 months, reported a $7.5 \%$ of failure at the SBRT site, without recording serious side-effects. However, the doses and the techniques adopted by Filippi et al. were different, either 3D-conformal radiotherapy or V-MAT were used (11). Yamamoto et al. treated 57 pulmonary metastases from 2001 to 2011 with SBRT (like Filippi et al., using various doses and techniques). They reported that CRC metastases were more radio-resistant than non-CRC metastases $(p=0.022)$ (12). Worse tumor control for pulmonary metastases from CRC was also reported by Oh $e t$ al. (20). Widder et al. compared outcomes in patients with lung metastases from CRC treated with SBRT or metastasectomy at the same institution. Although patients treated with SBRT had a worse prognosis (SBRT was proposed mainly for patients not suitable for surgery), survival after radiotherapy was not worse than that after surgery (14).

In the present study, all patients were treated with SBRT using the same technique (V-MAT) and a relatively small range of doses and fractionations. Not all treated lesions were the first site of recurrence; as a matter of fact, we did not consider overall survival as an endpoint. We only considered irradiated-site progression-free survival and systemic disease-free survival after SBRT. We found that after a median follow-up of 22.8 months, irradiated sitecontrol was $78.8 \%, 62.0 \%$ and $30.2 \%$ at 6,12 and 18 months, respectively. As expected, systemic disease-free survival was shorter than irradiated site progression-free survival. The clinical implication of this information could be that we should select patients with metastatic CRC who can benefit from local treatment considering not only the site to be treated but also the disease as a whole. This is particularly true when SBRT is considered in an attempt to affect the course of disease and in some selected case postpone the beginning of chemotherapy (21).

\section{Conclusion}

SBRT can be considered as local therapy in patients with lung metastases from CRC. However, before considering a patient suitable for SBRT, the planned outcome and the impact on systemic disease should be carefully investigated. Future prospective studies carried out on larger series are needed in order to select patients with lung metastases from CRC suitable for SBRT.

\section{References}

1 Siegel RL, Miller KD and Jemal A: Cancer statistics, 2015. CA Cancer J Clin 65: 5-29, 2015.

2 Mitry E, Guiu B, Cosconea S, Jooste V, Faivre J and Bouvier AM: Epidemiology, management and prognosis of colorectal cancer with lung metastases: a 30-year population-based study. Gut 59: 1383-1388, 2010.

3 Cunningham D, Atkin W, Lenz HJ, Lynch HT, Minsky B, Nordlinger B and Starling N: Colorectal Cancer. Lancet 375: 1030-1047, 2010.

4 Folprecht G, Grothey A, Alberts S, Raab HR and Kohne CH: Neoadjuvant treatment of unresectable colorectal liver metastases: correlation between tumour response and resection rates. Ann Oncol 16: 1311-1319, 2005.

5 Hellman S and Weichselbaum RR: Oligometastases. Journal of clinical oncology: official journal of the American Society of Clinical Oncology 13: 8-10, 1995.

6 Kaifi JT, Gusani NJ, Deshaies I, Kimchi ET, Reed MF, Mahraj $\mathrm{RP}$ and Staveley-O'Carroll KF: Indications and approach to surgical resection of lung metastases. J Surg Oncol 102: 187195, 2010.

7 Villeneuve PJ and Sundaresan RS: Surgical management of colorectal lung metastasis. Clin Colon Rectal Surg 22: 233-241, 2009.

8 Kim HJ, Kye BH, Lee JI, Lee SC, Lee YS, Lee IK, Kang WK, Cho HM, Moon SW and Oh ST: Surgical resection for lung metastases from colorectal cancer. J Korean Soc Coloproctol 26: 354-358, 2010.

9 Zelefsky MJ, Greco C, Motzer R, Magsanoc JM, Pei X, Lovelock M, Mechalakos J, Zatcky J, Fuks Z and Yamada Y: Tumor control outcomes after hypofractionated and single-dose stereotactic image-guided intensity-modulated radiotherapy for extracranial metastases from renal cell carcinoma. International journal of radiation oncology, biology, physics 82: 1744-1748, 2012.

10 Greco C, Zelefsky MJ, Lovelock M, Fuks Z, Hunt M, Rosenzweig K, Zatcky J, Kim B and Yamada Y: Predictors of local control after single-dose stereotactic image-guided intensity-modulated radiotherapy for extracranial metastases. Int J Radiat Oncol Biol Phys 79: 1151-1157, 2011.

11 Filippi AR, Badellino S, Ceccarelli M, Guarneri A, Franco P, Monagheddu C, Spadi R, Ragona R, Racca P and Ricardi U: Stereotactic ablative radiation therapy as first local therapy for lung oligometastases from colorectal cancer: a single-institution cohort study. Int J Radiat Oncol Biol Phys 91: 524-529, 2015.

12 Yamamoto T, Jingu K, Shirata Y, Koto M, Matsushita H, Sugawara T, Kubozono M, Umezawa R, Abe K, Kadoya N, Ishikawa Y, Kozumi M, Takahashi N, Takeda K and Takai Y: Outcomes after stereotactic body radiotherapy for lung tumors, with emphasis on comparison of primary lung cancer and metastatic lung tumors. BMC Cancer 14: 464, 2014.

13 Tree AC, Khoo VS, Eeles RA, Ahmed M, Dearnaley DP, Hawkins MA, Huddart RA, Nutting CM, Ostler PJ and van As NJ: Stereotactic body radiotherapy for oligometastases. Lancet Oncol 14: e28-37, 2013.

14 Widder J, Klinkenberg TJ, Ubbels JF, Wiegman EM, Groen HJ and Langendijk JA: Pulmonary oligometastases: metastasectomy or stereotactic ablative radiotherapy? Radiother Oncol 107: 409413, 2013.

15 Pasqualetti F, Panichi M, Sainato A, Matteucci F, Galli L, Cocuzza P, Ferrazza P, Coraggio G, Pasqualetti G, Derosa L, Sollini M, Mannelli L, Ortori S, Monzani F, Ricci S, Greco C, Fabrini MG and Erba PA: [(18)F]Choline PET/CT and stereotactic body radiotherapy on treatment decision making of oligometastatic prostate cancer patients: preliminary results. Radiat Oncol 11: 9, 2016. 
16 Berkovic P, De Meerleer G, Delrue L, Lambert B, Fonteyne V, Lumen N, Decaestecker K, Villeirs G, Vuye P, and Ost P: Salvage stereotactic body radiotherapy for patients with limited prostate cancer metastases: deferring androgen deprivation therapy. Clin Genitourin Cancer 11: 27-32, 2013.

17 Benedict SH, Yenice KM, Followill D, Galvin JM, Hinson W, Kavanagh B, Keall P, Lovelock M, Meeks S, Papiez L, Purdie T, Sadagopan R, Schell MC, Salter B, Schlesinger DJ, Shiu AS, Solberg T, Song DY, Stieber V, Timmerman R, Tome WA, Verellen D, Wang L, and Yin FF: Stereotactic body radiation therapy: the report of AAPM Task Group 101. Med Phys 37: 4078-4101, 2010.

18 US Department of Health and Human Services. Common terminology criteria for adverse events (CTCAE) version 4.02 . May 28, 2009. http://evs.nci.nih.gov/ftp1/CTCAE/Archive/ CTCAE_4.02_2009-09-15_QuickReference_8.5x11.pdf (accessed March 21, 2015).
19 Eisenhauer EA, Therasse P, Bogaerts J, Schwartz LH, Sargent D, Ford R, Dancey J, Arbuck S, Gwyther S, Mooney M, Rubinstein L, Shankar L, Dodd L, Kaplan R, Lacombe D, and Verweij J: New response evaluation criteria in solid tumours: revised RECIST guideline (version 1.1). Eur J Cancer 45: 228-247, 2009.

20 Oh D, Ahn YC, Seo JM, Shin EH, Park HC, Lim do H, and Pyo $\mathrm{H}$ : Potentially curative stereotactic body radiation therapy (SBRT) for single or oligometastasis to the lung. Acta oncologica 51: 596-602, 2012.

21 Wilson MK, Collyar D, Chingos DT, Friedlander M, Ho TW, Karakasis K, Kaye S, Parmar MK, Sydes MR, Tannock IF and Oza AM: Outcomes and endpoints in cancer trials: bridging the divide. Lancet Oncol 16: e43-52, 2015.

Received September 5, 2016

Revised September 29, 2016

Accepted November 21, 2016 